\title{
Dynamic Activation of Frontal, Parietal, and Sensory Regions Underlying Anticipatory Visual Spatial Attention
}

\author{
Gregory V. Simpson, ${ }^{1 \star}$ Darren L. Weber, ${ }^{1,2 \star}$ Corby L. Dale, ${ }^{1,3}$ Dimitrios Pantazis, ${ }^{4}$ Steven L. Bressler, ${ }^{5,6}$ \\ Richard M. Leahy, ${ }^{4}$ and Tracy L. Luks ${ }^{1}$ \\ ${ }^{1}$ Department of Radiology and Biomedical Imaging, University of California San Francisco, San Francisco, California 94143, ${ }^{2}$ Buck Institute for Age \\ Research, Novato, California 94945, ${ }^{3}$ Northern California Institute for Research and Education, San Francisco, California 94121, ${ }^{4}$ Department of Electrical \\ Engineering, University of Southern California, Los Angeles, California 90089, and ${ }^{5}$ Center for Complex Systems and Brain Sciences and ${ }^{6}$ Department of \\ Psychology, Florida Atlantic University, Boca Raton, Florida 33431
}

\begin{abstract}
Although it is well established that multiple frontal, parietal, and occipital regions in humans are involved in anticipatory deployment of visual spatial attention, less is known about the electrophysiological signals in each region across multiple subsecond periods of attentional deployment. We used MEG measures of cortical stimulus-locked, signal-averaged (event-related field) activity during a task in which a symbolic cue directed covert attention to the relevant location on each trial. Direction-specific attention effects occurred in different cortical regions for each of multiple time periods during the delay between the cue and imperative stimulus. A sequence of activation from V1/V2 to extrastriate, parietal, and frontal regions occurred within $110 \mathrm{~ms}$ after cue, possibly related to extraction of cue meaning. Direction-specific activations $\sim 300 \mathrm{~ms}$ after cue in frontal eye field (FEF), lateral intraparietal area (LIP), and cuneus support early covert targeting of the cued location. This was followed by coactivation of a frontal-parietal system [superior frontal gyrus (SFG), middle frontal gyrus (MFG), LIP, anterior intraparietal sulcus (IPSa)] that may coordinate the transition from targeting the cued location to sustained deployment of attention to both space and feature in the last period. The last period involved direction-specific activity in parietal regions and both dorsal and ventral sensory regions [LIP, IPSa, ventral IPS, lateral occipital region, and fusiform gyrus], which was accompanied by activation that was not direction specific in right hemisphere frontal regions (FEF, SFG, MFG). Behavioral performance corresponded with the magnitude of attention-related activity in different brain regions at each time period during deployment. The results add to the emerging electrophysiological characterization of different cortical networks that operate during anticipatory deployment of visual spatial attention.
\end{abstract}

\section{Introduction}

The ability to covertly shift and hold anticipatory spatial attention is an important aspect of cognition (Posner 1980). Functional MRI studies have shown that multiple frontal, parietal, and visual sensory regions are active during anticipatory deployment of visual spatial attention (Kastner and Ungerleider, 2000; Corbetta and Shulman 2002; Serences and Yantis, 2006). Scalp ERP studies indicate that multiple direction-specific attentional stages occur within fractions of a second following a cue to covertly deploy attention to a spatial location in anticipation of a stimulus, and they support the possibility of different configurations of

Received March 24, 2010; revised May 24, 2011; accepted June 15, 2011.

Author contributions: G.V.S., C.L.D., and T.L.L. designed research;D.L.W. and C.L.D. performed research; G.V.S., D.L.W., D.P., S.L.B., and R.M.L. analyzed data; G.V.S., C.L.D., S.L.B., R.M.L., and T.L.L. wrote the paper.

This work was supported by NIH NINDS Grants R01 NS27900 and R01 NS45171 (G.V.S.), National Institute of Biomedical Imaging and Bioengineering Grants R01 EB002010 and R01EB009048 (R.M.L.), NIMH Grant 64204 (S.L.B.), and NIA Grant K01AG027369 (T.L.L.). We thank Drs. John Mosher, Sylvain Ballet, Thomas Ferree, Matti Hamalainen, Thomas Nichols, John Kornak, and Morgan Hough for helpful suggestions, statistical advice, and software developments, and Eleanor Wong for technical assistance.

*G.V.S. and D.L.W. contributed equally to this work.

Correspondence should be addressed to Dr. Gregory V. Simpson at his present address, Brain Plasticity Institute, 1 Montgomery Street, 7th Floor, San Francisco, CA 94104. E-mail: Greg.BrainPlasciticityInstitute@gmail.com.

DOI:10.1523/JNEUROSCI.1519-10.2011

Copyright $\odot 2011$ the authors $\quad 0270-6474 / 11 / 3113880-10 \$ 15.00 / 0$ active cortical regions in each deployment stage, although the brain regions involved in each ERP stage are not well established (Hopf and Mangun 2000; Nobre et al., 2000a; Praamstra et al., 2005; Slagter et al., 2005; van der Lubbe et al., 2006; Dale et al., 2008).

While there is general support for top-down frontal and parietal control during visual attention (Buschman and Miller, 2007; Saalmann et al., 2007; Bressler et al., 2008; Ruff et al., 2008; Capotosto et al., 2009; Gregoriou et al., 2009), evidence for the notion that different frontal and parietal control regions are involved during the different temporal stages of anticipatory attentional deployment is just beginning to emerge (Green and McDonald, 2008; Lauritzen et al., 2009; Pantazis et al., 2009). Recent EEG/ MEG imaging studies of oscillatory activity provided initial insights into the complexities of the dynamic electrophysiological activity in brain regions during attentional deployment. Different distributions of active cortical regions have been found for alpha, beta, and gamma frequencies when measured over the whole delay period (Siegel et al., 2008). Siegel et al. (2008) examined the dynamics for two of these active regions and found that the temporal pattern of direction-specific attention activity within each region, and the relative timing between regions, also varied with frequency. Separate investigations of dynamics have been conducted for theta (Green and McDonald, 2008) and alpha band 


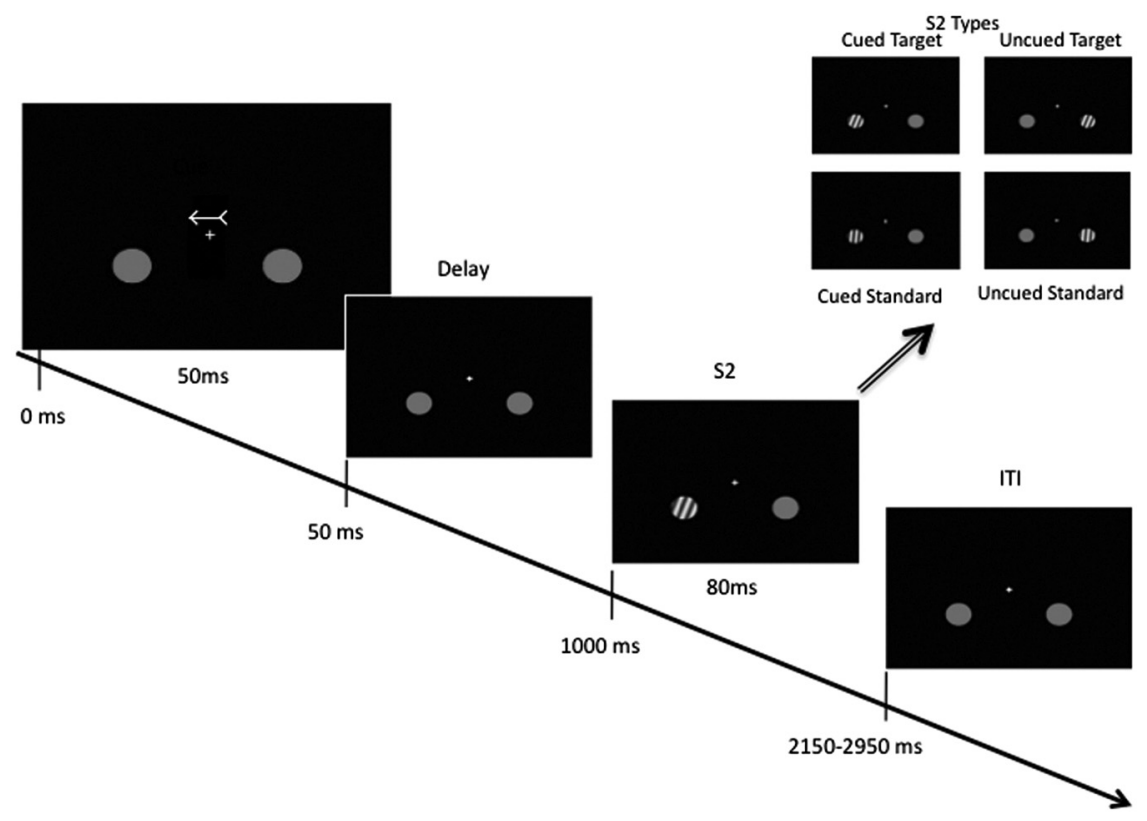

Figure 1. Task design: covert anticipatory shifts of visual spatial attention. On each trial, a central arrow cue ( $50 \mathrm{~ms}$ duration) directed subjects to covertly deploy and hold their attention to the left $(\longleftarrow$ ) or right $(\hookrightarrow)$ lower visual quadrant (continuously marked by circular gray patches). Following a $1 \mathrm{~s}$ delay, a second stimulus ( $\$ 2,80 \mathrm{~ms}$ duration) was delivered at either the cued or uncued location (50/50 probability of occurring at either location). Subjects performed a go/no-go discrimination of the 52 grating orientation only if it occurred at the cued location and ignored any stimuli presented at the uncued location. The $\$ 2$ stimuli were categorized into four types: (cued vs uncued location) by (target orientation vs standard orientation). $\mathrm{A} 5$ or $20^{\circ}$ clockwise tilt of the grating orientation from vertical constituted a standard or target feature, respectively. The design produced an equal probability of receiving a relevant stimulus (i.e., $S 2$ at the cued location) or an irrelevant distractor (i.e., $S 2$ at the uncued location) and required performance of a feature discrimination at the relevant spatial location. The size of the arrow cue is larger, and the contrast level of gratings is exaggerated for easier visualization.

(Pantazis et al., 2009) activity, revealing that cortical areas with attention-related activity vary at each period during deployment. Comparison of these studies suggests that while there are commonalities, the active cortical regions at each stage differ for alpha and theta. Detailed determinations of which regions are involved at each period, and the nature of the electrophysiological processes in each region, is important for a full understanding of the functional properties of attentional deployment. The present study measures stimulus-locked, signal-averaged [event-related field (ERF)] direction-specific attention activity across multiple frontal, parietal, and sensory cortical regions at each of multiple time periods during deployment. We examined whether active cortical regions support an early covert shift to the cued location, similar to that reported in visual search (Buschman and Miller, 2007), and a late period that may provide a top-down controlled bias signal for the location and feature of the anticipated target. We also propose that between the early and late periods, there is coordination between frontal and parietal regions to make the transition from early spatial orienting to biasing of both space and feature.

\section{Materials and Methods \\ Participants}

Eight right-handed, neurologically normal volunteers (six male; age, 27.4 years; SD, 4.3 years) participated in the study. Three additional subjects were excluded due to excessive head movements $(>5 \mathrm{~mm}$ ) during MEG recording. Written informed consent was obtained from all subjects, and the experiment was approved by the Institutional Review Board of the University of California, San Francisco.

Task and stimuli

Subjects were instructed to maintain fixation on a cross-hair located at the center of their visual field (Fig. 1). They performed a cued visual spatial attention task while their MEG was recorded. A brief central arrow cue $(50 \mathrm{~ms})$ instructed subjects to covertly deploy and hold their attention to the left or right lower quadrant in anticipation of a unilateral S2 stimulus to be presented $1 \mathrm{~s}$ later at either the cued or uncued location (50/50 probability of occurring at either location); that is, the cue was not predictive of S2 spatial location, but was $100 \%$ instructive regarding where to attend. If the S2 occurred at the cued location, subjects performed a go/no-go grating discrimination; if it occurred at the uncued location, it was to be ignored. The $\mathrm{S} 2$ gratings could be one of two types: a $5^{\circ}$ tilt clockwise from vertical was a nontarget requiring no response, and a $20^{\circ}$ tilt was a target requiring a button press with the right index finger (if it occurred at the cued location). This produced a $25 \%$ probability that the S2 would be a target stimulus at the cued location, thus requiring a response. The design produced an equal probability of receiving a relevant stimulus (i.e., S2 at the cued location) or an irrelevant distractor (i.e., S2 at the uncued location), and required performance of a feature discrimination at the relevant spatial location.

The locations of the anticipated S2 stimuli were continuously marked by circular gray patches $(50 \%$ greater luminance relative to background), $2.25^{\circ}$ in diameter located in the left and right lower quadrants at $5.125^{\circ}$ eccentricity along the diagonal from fixation to the center of the patch. The $\mathrm{S} 2$ grating stimuli were grayscale images of stationary sine-wave-modulated contrasts at 1.75 cycles per degree that filled the left or right circular patch for a duration of $85 \mathrm{~ms}$. The peak-to-peak contrast modulation was from $10 \%$ above background luminance to $90 \%$ above background; consequently, the mean luminance was the same as the nonmodulated gray patch, i.e., $50 \%$ above background, thereby not creating a luminance change with the S2 onsets. The nontarget gratings were rotated clockwise from vertical by $5^{\circ}$, and the target gratings were rotated by $25^{\circ}$. This ensured that subjects could not determine whether the S2 was a target stimulus simply by detecting that it was off the vertical; they had to determine the degree of tilt from vertical. The discrimination difficulty resulted in sufficient variation in performance within and between subjects to conduct analyses of the relationship between performance and magnitude of anticipatory attention effects. Arrow cues $\left(\leftarrow<\right.$ and $>\rightarrow$ ) were $0.75^{\circ}$ wide and $0.50^{\circ}$ high, and were presented immediately above the fixation cross. The arrow cue was designed to convey directional information as a unitary entity. Both sides of the cue object convey the same directional information. For example, in a left cue $(\leftarrow<)$ both the left and right sides convey the same directional meaning $(\leftarrow$ and $-<$ ). Subjects reported that the cue is processed as a unitary entity, an arrow, that conveys directional information seemingly "automatically" (consistent with the literature on reflexive processing of arrows; Ristic et al., 2002). We used an arrow cue, because we wanted an unambiguous, highly overlearned and extremely rapidly processed cue to examine the possibility of rapid shifting. There was a $2150-2950 \mathrm{~ms}$ variable intertrial interval. Each trial consisted of an arrow cue (left/right direction was varied pseudorandomly across trials) followed $1 \mathrm{~s}$ later by an S2 stimulus, followed by a variable intertrial interval. Participants received 18 blocks of 50 trials per block ( 900 trials total), with each block lasting $\sim 130$ s and breaks between blocks as needed to sustain good attention throughout the study. Stimuli were delivered via a liquid crystal projection system with a screen placed $36 \mathrm{~cm}$ from the nasion. 


\section{Data collection}

MRI acquisition. Anatomical MRIs were obtained for each participant to generate models of cerebral tissues for MEG source imaging and definition of cortical regions of interest (ROIs). The MRI data were acquired at 1.5 tesla, using a SENSE head coil (Philips Gyroscan Intera; Philips Medical Systems). A whole head volume was acquired in 200 axial slices with a three-dimensional gradient echo sequence $(256 \times 256$ matrix; $1.0 \times 1.0 \times 1.2 \mathrm{~mm}$; turbo factor, 180; shot interval, $3000 \mathrm{~ms}$; TI, 769.6 ms; TE, $3.7 \mathrm{~ms}$; TR, $7.9 \mathrm{~ms}$; flip angle, $8^{\circ}$ ). For spatial coregistration of MRI data with the MEG sensor array, we marked the nasion and the left and right preauricular points using multimodality radiographic markers (MM 3002; IZI Medical Products).

$M E G$ acquisition and preprocessing. Continuous MEG signals were acquired using a 275channel whole-cortex CTF Omega 2000 system (VSMedTech Systems). A third-order synthetic gradiometer correction was applied to remove far-field noise. The head location within the MEG helmet was recorded with three magnetic coils during the short breaks between blocks, and subjects with excessive head movements $(>5 \mathrm{~mm}$ ) were excluded from analyses. The MEG recording bandpass was $0-300 \mathrm{~Hz}$, and data were sampled at 1200

$\mathrm{Hz}$. Vertical and horizontal eye movements were measured with bipolar vertical and bipolar horizontal DC-recorded electroculograms. All trials were inspected for eye movements and other artifacts (MEG artifacts, muscle activity, eye blinks, etc.) and contaminated trials were discarded, leaving $\sim 700$ trials per subject for analyses. The artifact-rejected MEG data were low-passed filtered at $40 \mathrm{~Hz}$ and averaged, using epochs from -200 to 1400 $\mathrm{ms}$, with respect to cue onset to create ERFs for source imaging.

\section{Data processing}

MEG cortical source imaging. A tessellated representation (40,000 vertices) of the cortical surface was constructed from the volumetric T1-weighted MR images of each subject, coregistered to the MEG data. The MR images were segmented using FreeSurfer (Dale et al., 1999). The spatial distributions of signal-averaged sensor waveforms (ERFs) were mapped to the cortical surface of each subject using a regularized L2 minimum norm inverse procedure (Hämäläinen et al., 1993; Dale et al., 2000; Darvas et al., 2004), with thepubliclyavailableBrainStormMatlabtoolbox(http://neuroimage. usc.edu/brainstorm/). The resulting map provides an estimate of cortical current density at every vertex for each time sample of each ERF. The cortical current magnitudes at successive time points during the experimental epoch (brain source waveforms) provide the dynamic activity information for further analyses.

Extraction of ROI-based MEG data. We analyzed cortical current image maps in each subject using anatomically predefined ROIs. This a priori approach is desirable for its data reduction and its signal detection sensitivity because it summarizes activity over the vertices in each ROI and can detect weak brain activation that could be missed with methods that examine all vertices in the brain requiring severe statistical thresholding. We defined 11 regions commonly found across multiple fMRI and PET studies of visual spatial attention (Corbetta et al., 1998; Coull and Nobre, 1998; Gitelman et al., 1999; Kastner et al., 1999; Rosen et al., 1999; Hopfinger et al., 2000; Nobre et al., 2000b; Vandenberghe et al., 2000; Yantis et al., 2002; Molenberghs et al., 2007; Serences and Yantis, 2007). Each ROI was defined by deriving the superset of published Talairach coordinates for that region and determining the maximum range of values reported across studies in $x, y$, and $z$ axes. The ROI locations and extents are shown in Figure 2, and the Talairach coordinates for the center of each ROI are given in Table 1. The Talairach coordinates of ROIs were mapped across individual subject's brains using surface-based morphing
Table 1. Talairach coordinates for ROls

\begin{tabular}{|c|c|c|c|c|c|c|}
\hline \multirow[b]{2}{*}{ ROI } & \multicolumn{3}{|c|}{ Left hemisphere } & \multicolumn{3}{|c|}{ Right hemisphere } \\
\hline & $x$ & $y$ & $z$ & $x$ & $y$ & $z$ \\
\hline \multicolumn{7}{|l|}{ Frontal lobe } \\
\hline MFG & -42 & 38 & 24 & 47 & 38 & 25 \\
\hline FEF & -43 & -5 & 48 & 38 & -3 & 52 \\
\hline SFG & -17 & 52 & 34 & 17 & 50 & 39 \\
\hline \multicolumn{7}{|l|}{ Parietal lobe } \\
\hline IPL & -54 & -51 & 34 & 54 & -48 & 27 \\
\hline IPSa & -27 & -53 & 48 & 26 & -56 & 52 \\
\hline IPSV & -26 & -78 & 26 & 25 & -75 & 26 \\
\hline LIP & -29 & -66 & 45 & 31 & -71 & 45 \\
\hline \multicolumn{7}{|l|}{ Occipital lobe } \\
\hline Fusiform & -35 & -78 & -8 & 33 & -76 & -9 \\
\hline LO & -46 & -84 & 2 & 46 & -83 & 7 \\
\hline Cuneus & -34 & -90 & 16 & 36 & -86 & 18 \\
\hline V1/V2 & -14 & -78 & 12 & 15 & 69 & 8 \\
\hline
\end{tabular}

in FreeSurfer (Fischl et al., 1999a,b). We use relatively large ROIs (in keeping with the blurred nature of MEG images) that provide approximate locations of activity, which can be referred to the growing functional imaging literature for comparison. Due to the relatively low spatial resolution of MEG imaging, activity found in an ROI does not pinpoint the activity to that ROI nor restrict it to that ROI, but indicates that the activity arises in the vicinity of that ROI. The same interpretation issue applies to a data-driven MEG analysis approach because the results are also spatially blurred.

Each ROI contains multiple vertices from the tessellated cortical surface. To reduce the data and obtain a representative waveform for each ROI, we extracted the source waveforms for all vertices within each ROI, identified the 20 vertices having the largest peak amplitude, and derived the mean waveform of these 20 vertices, using the same vertices for every time point, thereby reducing the data to a single waveform representation for each ROI. A representative waveform was derived for each condition (cue left, cue right) in each of the 11 ROIs for the cue delay period (including a $200 \mathrm{~ms}$ baseline preceding the onset of the cue and $1000 \mathrm{~ms}$ after cue onset), and these were submitted to statistical analyses (see below, Analyses). 
MEG cortical cross-talk and interpretive issues. The cortical images of activity are spatially blurred due to the nature of the MEG inverse method. This results in spread of activity from one region into a neighboring region (cross talk), and the extent of spread depends upon the point-spread function in the area. Regions that are further apart will have less contamination from activity arising in the other area. While some of the regions used in this study are relatively close to each other, it should be noted that it is possible to detect activity arising in one region versus that of its neighbor when their activity differs in timing or in response to experimental conditions, thereby overcoming the common activity in each region (as is found in the present data).

Temporal definition of time periods used during cue-target delay. To further reduce the high dimensionality of the data and speak directly to the large ERP literature, we defined four a priori time periods corresponding to commonly reported scalp ERP components during the delay following a cue. We defined the time periods, analogous to defining "components," through an assessment of the range of postcue latencies reported in the literature for the following four attention-related scalp components (Harter et al., 1989; Hopf and Mangun, 2000; Nobre et al., 2000a; van Velzen et al., 2002; Praamstra et al., 2005; Slagter et al., 2005; Green and McDonald, 2006, 2010; Jongen et al., 2006; Simpson et al., 2006; van der Lubbe et al., 2006; Grent-' $t$-Jong and Woldorff, 2007; Dale et al., 2008). (1) An early, short-duration process (positivity contralateral early) occurring during 125-175 ms; (2) a subsequent component, often referred to as the early directing attention negativity (EDAN), occurring between 250 and $350 \mathrm{~ms}$; (3) a middle period associated with activity commonly called the anterior directing attention negativity (ADAN), occurring between 400 and $500 \mathrm{~ms}$; and (4) a late period toward the end of the cue delay up to the onset of the target, which would correspond to $800-1000 \mathrm{~ms}$ in this study, often called the late attention directing positivity. Because the latency and duration of components varies somewhat across published studies within our a priori ranges, we divided each period into two equal duration windows to better define the timing of the component activity in the present study, resulting in eight windows.

\section{Data analyses}

Direction-specific anticipatory attention activity during attentional deployment. We defined direction-specific attention activity as the difference in activity in a region when anticipatory attention is directed to the contralateral versus ipsilateral visual field location. Our hypothesis was that underlying each period during attentional deployment there is directionspecific attention activity (cue direction by hemisphere interaction) in a subset of cortical regions (ROIs). This was assessed by first submitting the magnitude of MEG cortical ROI activity to an ANOVA having four within-subject factors-cue direction (left, right), cortical hemisphere (left, right), cortical region (ROI), and temporal component (components) - and testing for a significant cue by hemisphere by ROI by component interaction. Finding this four-way interaction to be significant $\left(F_{(60,420)}=2.734 ; p=0.04\right)$ after Greenhouse-Geisser correction for sphericity, we then examined each of the components (time periods) and tested for significant cue by hemisphere interactions in each ROI without corrections for multiple comparisons (Fig. 3) and report these interaction effects in the Results section.

Correspondence between anticipatory attention activity and subsequent performance. To determine whether anticipatory attentional modulations of cortical activity benefit subsequent behavioral performance, we conducted an analysis of the magnitude of attentional modulation in each cortical region versus the speed of reaction time (RT). This was accomplished by dividing trials into two groups: the fast group (trials with RTs less than the median RT) and the slow group (trials with RTs greater than the median RT). An ERF (the mean across trials) was obtained for each group of trials (fast and slow). A direction-specific attention modulation (the between-condition difference in ERF magnitude for attention cued to the contralateral hemifield vs ipsilateral hemifield) was calculated for fast and slow ERFs in each cortical region. The attention modulation values were entered into an ANOVA with two withinsubjects factors: RT (fast, slow) and hemisphere (left, right). An ANOVA was performed for each of the four time windows used in the main analyses above.

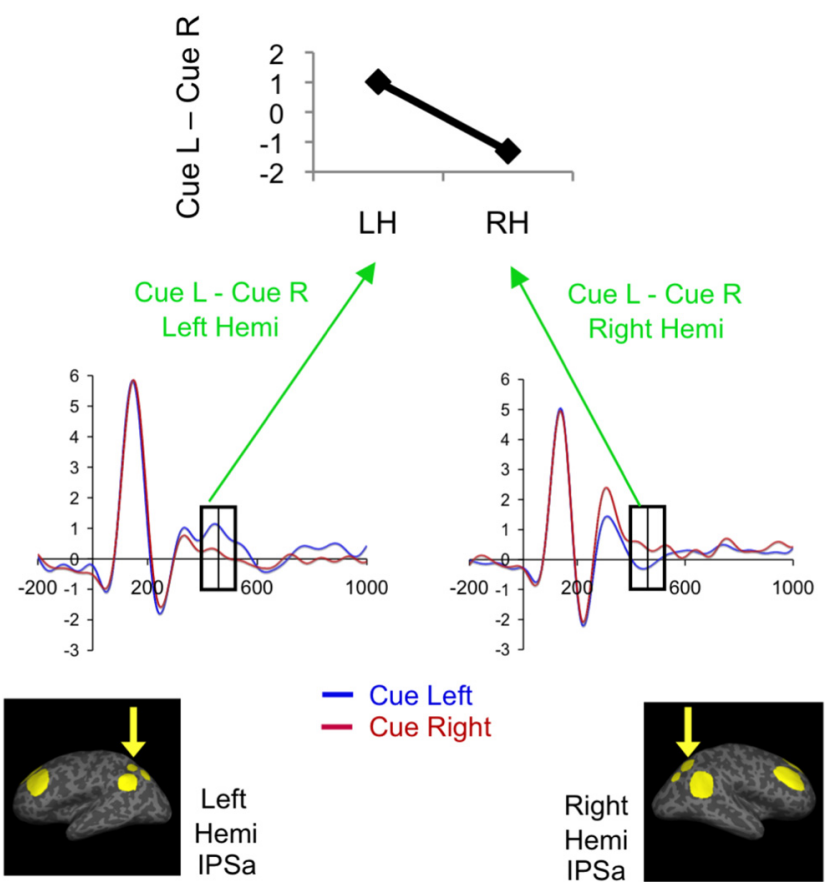

Figure 3. Method for deriving direction-specific anticipatory visual attention effects. Direction-specific attention effects on brain activity are defined in the ANOVA model as interaction effects between factors cue direction (left, right) and R0I-hemisphere (left, right). An example of such an interaction is graphed at the top. ROl activity is measured in the left and right hemisphere under two conditions: when spatial attention is cued toward or away from the visual field contralateral to the ROI hemisphere, corresponding to conditions in which the spatial location contralateral to the cortical region is attended or unattended, respectively. For example in the right hemisphere ROl, cue left-cue right conditions correspond to attendedunattended conditions. Comparison between the same conditions (cue left- cue right) for the left hemisphere should reveal the effect with opposite sign because this comparison corresponds to unattended-attended conditions. This interaction effect appears graphically as a nonzero slope between the values for the left and right hemispheres. An example is illustrated in the figure for IPSa. The IPSa MEG activity waveforms for each condition (cue left and cue right) are plotted in the middle. The a priori time window for defining the amplitudes entered into the ANOVA ( $400-500 \mathrm{~ms}$ in this example) is shown by the vertical box. (Note that to capture the activity predicted by the literature, each a priori time window is divided into two equal subwindows due to variance in the latencies from the literature. The data shown in the plot in the top panel are for the $400-450 \mathrm{~ms}$ subwindow.) The mean amplitude during the subwindow is determined for each attention condition, and the resultant value for subtracting cue left and cue right mean amplitudes for a hemisphere is plotted in the top panel, reflecting the attention effect in that hemisphere.

\section{Results}

\section{Behavioral results}

Participants were required to respond only to target gratings presented at the cued location. Participants correctly detected about $80 \%$ of cued targets $[81.5 \pm 11.4 \%$; the false negative rate was about $17 \%(17.3 \pm 11.1 \%)]$ with $<2 \%$ false positive responses $(1.6 \pm 1.8 \%)$. The target detection rate was similar for the left visual field $(79.8 \pm 14.2 \%)$ and right visual field $(83.2 \pm 10.4 \%$; $t_{(7)}=-0.92$, not significant). The mean reaction time for cued targets was $500.2 \pm 79.2 \mathrm{~ms}$. The reaction time for cued targets was significantly faster by about $20 \mathrm{~ms}$ for the right visual field $(490.4 \pm 80.7 \mathrm{~ms})$ than for the left visual field $(510.7 \pm 79.9 \mathrm{~ms}$; $\left.t_{(7)}=3.48 ; p<0.05\right)$.

\section{Flow of cortical activations preceding earliest (150-175 ms after cue) direction-specific attention effect}

It is widely postulated that cue-driven voluntary deployment of spatial attention must proceed via bottom-up extraction of sym- 


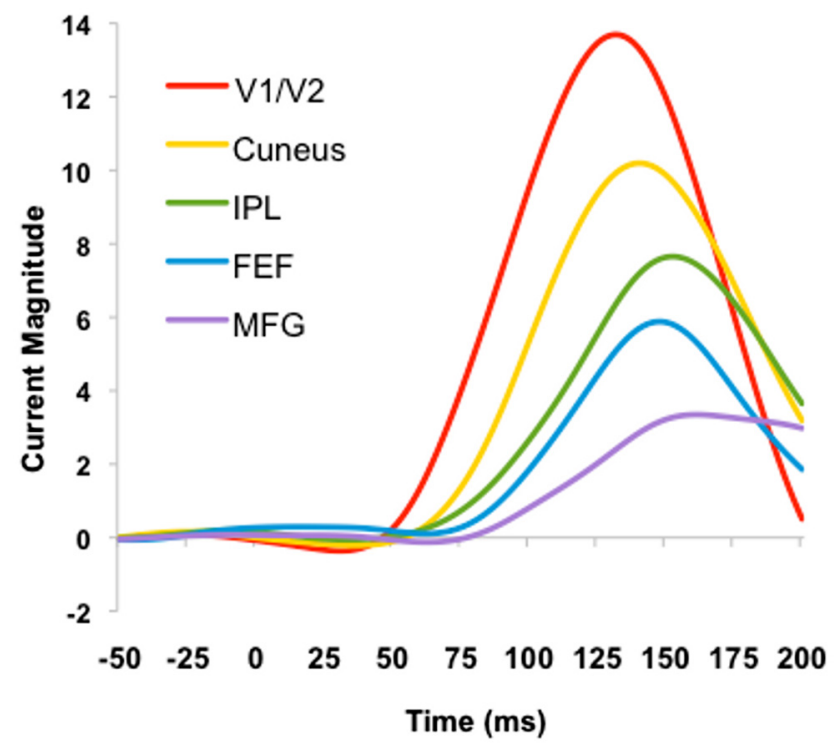

Figure 4. Early onsets of activity across cortical regions. The waveforms illustrate the initial onsets of activity to the cue stimulus from representative ROIs in occipital (V1/V2; cuneus), parietal (IPL), and frontal lobes (FEF, MFG). The waveforms show differences in onset timing consistent with conduction time between regions and indicate that bottom-up processing of cue information has occurred throughout these regions by $\sim 110 \mathrm{~ms}$ after cue.

bolic cue meaning that leads to top-down direction-specific attentional deployment processes. Yet, human cueing studies have not examined the initial temporal flow of activations from lowertier visual regions to parietal and frontal control regions to determine whether control regions are activated sufficiently in advance of the first direction-specific attention effect to allow for communication between control regions and the sensory region that is modulated. To determine this, we performed an analysis of the first onset latencies in occipital, parietal, and frontal regions before the first attention effects in sensory cortex (during 150$175 \mathrm{~ms}$ in cuneus; see below). For each cortical region, the onset of event-related activity to cues was estimated as the first time point at which the magnitude exceeded a baseline threshold (i.e., the mean plus two SDs of activity occurring in the baseline period defined as -200 to $+25 \mathrm{~ms}$ relative to cue onset). The sequence of cortical onset latencies is as follows: V1/V2, $66.5 \mathrm{~ms}$; cuneus, 77.2 $\mathrm{ms}$; inferior parietal lobe (IPL), $84.4 \mathrm{~ms}$; frontal eye field (FEF), $94.8 \mathrm{~ms}$; middle frontal gyrus (MFG), $107.3 \mathrm{~ms}$. The results reveal a sequence of activations from sensory to parietal to frontal regions (Fig. 4) between 60 and $107 \mathrm{~ms}$, indicating that there had been significant activation of sensory, parietal, and frontal regions at least $40 \mathrm{~ms}$ before the first direction-specific effect observed at $150 \mathrm{~ms}$ (see the following section for direction-specific latencies).

Examination of multiple temporal periods during anticipatory deployment of visual spatial attention: direction-specific attention effects

Earliest direction-specific modulation of sensory cortex

Following the bilateral non-direction-specific activations described above, there was a statistically significant and directionspecific modulation of the cuneus (Fig. 5) in which the activity was larger in the hemisphere contralateral to the location indicated by the cue [cue by hemisphere interaction during 150-175 ms after cue, $F_{(1,7)}=12.92, p=0.009$; post hoc analyses for cue effect (cue left vs cue right) within hemisphere, left hemisphere

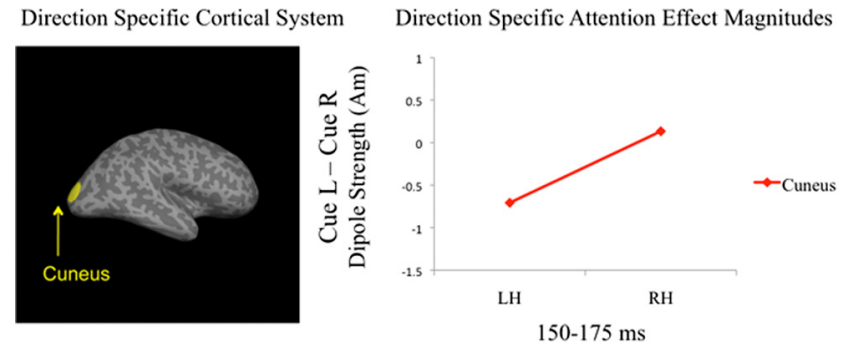

Figure 5. Earliest (150-175 ms) direction-specific effect. The first statistically significant direction-specific attentional modulation of cortex was found in the occipital cuneus region during the $150-175$ ms period following the cue. The cue by hemisphere interaction effect in the cuneus is illustrated in the right panel: the difference in MEG magnitude between the two attention conditions (cue left - cue right) is shown for the LH and RH cuneus regions.

$(\mathrm{LH}), t=2.64, p<0.05$; right hemisphere $(\mathrm{RH})$, not significant]. No other direction-specific effects were found during this period.

Early period direction-specific attention effects in parietal and occipital regions

During the next temporal period (250-350 ms after cue), significant direction-specific effects were found in both parietal [lateral intraparietal area (LIP)] and occipital (cuneus) regions as illustrated in Figure 6 (cue by hemisphere interaction, LIP, 250-300 $\mathrm{ms}, F_{(1,7)}=7.29, p=0.03$; cue effect within $\mathrm{RH}, t=2.89, p<$ $0.05 ; 300-350 \mathrm{~ms}, F_{(1,7)}=8.95, p=0.02$; cue effect within LH, $t=2.05, p<0.05$; cuneus, $250-300 \mathrm{~ms}, F_{(1,7)}=8.36, p=0.02$, bilateral). There was a trend for cue by hemisphere interaction in the lateral occipital $(\mathrm{LO})$ region $\left(250-300 \mathrm{~ms}, F_{(1,7)}=5.49\right.$, $p=0.052$ ).

\section{Middle period direction-specific effects in prefrontal and parietal} control regions

In contrast to the preceding periods (and the last period, see below), direction-specific effects during the middle period (400$500 \mathrm{~ms}$ after cue) involved prefrontal [superior frontal gyrus (SFG), MFG] and parietal [LIP, anterior intraparietal sulcus (IPSa), IPL] control regions without any directional effects in sensory regions (Fig. 7). Cue by hemisphere interactions were found in the SFG $\left(400-450 \mathrm{~ms}, F_{(1,7)}=6.83, p=0.03\right.$, bilateral) and the MFG (450-500 ms, $F_{(1,7)}=7.70, p=0.03$, bilateral), as well as the LIP (400-450 ms, $F_{(1,7)}=10.69, p=0.01$, bilateral), IPSa (400-450 ms, $F_{(1,7)}=12.56, p=0.009$, bilateral; $450-500$ $\mathrm{ms}, F_{(1,7)}=16.06, p=0.005$; cue effect within the LH, $t=3.33$, $p<0.01)$, and IPL $\left(400-450 \mathrm{~ms}, F_{(1,7)}=16.88, p=0.005\right.$; cue effect within the RH, $t=2.05, p<0.05$ ).

Late sustained period: direction-specific effects in different parietal and occipital regions from the early period

The final period during anticipatory deployment $(800-1000 \mathrm{~ms}$ after cue) involves direction-specific effects in a fourth set of cortical regions that is different in its cortical configuration from the earlier periods. Significant cue by hemisphere effects were found in LIP, IPSa, and ventral IPS (IPSv) (Fig. 8). In contrast to preceding periods having modulation only in dorsal occipital regions, significant cue by hemisphere effects were found in both dorsal (LO) and ventral (fusiform) occipital regions, consistent with attention-related modulation based on both location and feature aspects of the anticipated target (LIP, 800-900 ms, $F_{(1,7)}=$ 11.66, $p=0.01$; cue effect within LH, $t=2.89, p<0.01$; 900 $1000 \mathrm{~ms}, F_{(1,7)}=13.76, p=0.008$, cue effect within LH, $t=4.9$, $p<0.001$; IPSa, 800-900 ms, $F_{(1,7)}=6.44, p=0.04$, cue effect within LH, $t=3.03, p<0.01 ; 900-1000 \mathrm{~ms}, F_{(1,7)}=6.51, p=$ 0.04 , cue effect within LH, $t=2.55, p<0.05$; IPSv, $800-900 \mathrm{~ms}$, 
Direction Specific Cortical System
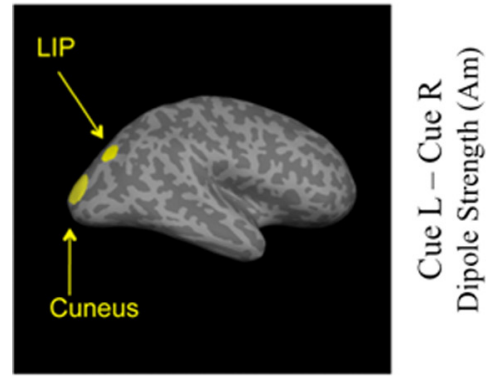

Direction Specific Attention Effect Magnitudes

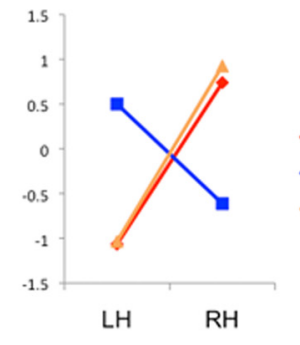

$250-300 \mathrm{~ms}$

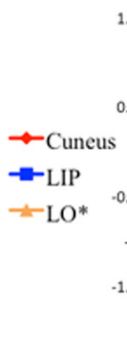

300-350 ms

Figure 6. Early period ( $250-350 \mathrm{~ms}$ ): direction-specific attention system. The early period, $250-350 \mathrm{~ms}$ after cue, significant direction-specific attention effects occurred in a parietal and occipital system involving LIP and cuneus regions. In addition, there was a trend effect in the LO region ( $p=0.052$ ) shown in the plots of attention effects. The cue by hemisphere attention effects are illustrated in the right panels for the subwindows of the early period ( $250-300$ and $300-350 \mathrm{~ms}$ ).

Direction Specific Cortical System

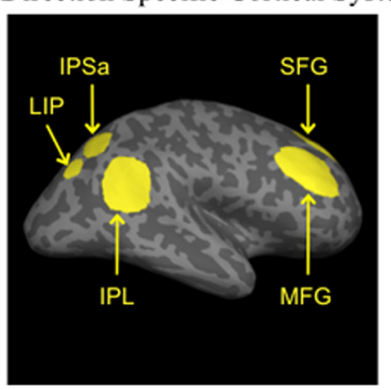

\section{Direction Specific Attention Effect Magnitudes}

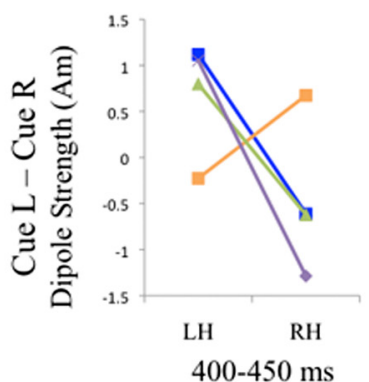

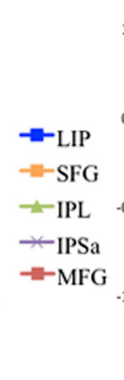

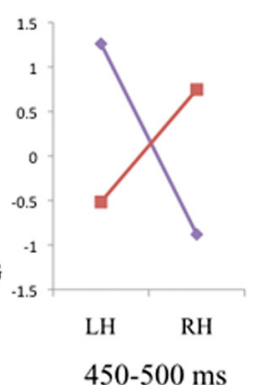

Figure 7. Middle period (400-500 ms): direction-specific attention system. Direction-specific processing in the middle period (400-500 ms after cue) during anticipatory attentional deployment occurred in a different system involving prefrontal and parietal control regions — SFG, MFG, LIP and IPSa — and did not involve sensory regions.

$F_{(1,7)}=7.90, p=0.03$, bilateral; 900-1000 ms, $F_{(1,7)}=6.80, p=$ 0.04 , bilateral; LO region, $900-1000 \mathrm{~ms}, F_{(1,7)}=7.08, p=0.03$, cue effect within LH, $t=3.4, p<0.01$; fusiform, $800-900 \mathrm{~ms}, F_{(1,7)}=$ 5.87, $p=0.046$, bilateral; $900-1000 \mathrm{~ms}, F_{(1,7)}=6.20, p=0.042$, bilateral).

\section{Late sustained activity in frontal regions}

The direction-specific (cue by hemisphere) effects during the late sustained period occurred only in parietal and occipital regions and not in frontal regions. Frontal areas are commonly found in fMRI studies of anticipatory attention, and control regions can play a more general role that does not reflect a direction-specific effect. To determine whether frontal regions might be active at the same time that there were direction-specific activations in the parietal and occipital regions, we tested for significant increases in activity above baseline during the late window (800-1000 ms) for the three frontal regions (FEF, SFG, MFG). This was accomplished for each frontal region (FEF, SFG, MFG) by deriving the mean of the absolute value of activity during each of the two windows in the period (800-900 and 900$1000 \mathrm{~ms}$ ) and obtaining the average of the two conditions (cue left, cue right) in each subject. We then tested whether the activity in each window was significantly greater than the mean of the 200 ms precue baseline window using a one-tailed $t$ test [one-tailed because the postcue absolute value activity can only be greater (it cannot be smaller) than the mean of 0 of the baseline period]. There was significantly increased activity in FEF, SFG, and MFG in the right hemisphere (FEF, 800-900 ms, $t_{(7)}=-3.89, p<$ $0.01 ; 900-1000 \mathrm{~ms}, t_{(7)}=-3.43, p<0.05$; SFG, $800-900 \mathrm{~ms}$, $t_{(7)}=-5.54, p<0.001 ; 900-1000 \mathrm{~ms}$, $t_{(7)}=-6.33, p<0.001 ; \mathrm{MFG}, 800-900$ $\mathrm{ms}, t_{(7)}=-4.12, p<0.01 ; 900-1000$ $\left.\mathrm{ms}, t_{(7)}=-3.71, p<0.01\right)$, and not in the left hemisphere (see Fig. 8).

Confirmation of a priori time periods $s$ as representative time periods

The above set of results report small variations in onset or offset latency within the a priori time periods, consistent with the amount of temporal variation reported in the EEG literature (i.e., significant effects occurred in the first or second window or spanned both windows in the a priori period). To provide a qualitative assessment that the literature-based a priori windows are representative of the time periods during which there are significant direction-specific attention effects, we ran an exploratory ANOVA on successive windows across the epoch ( $25 \mathrm{~ms}$ windows for $100-200 \mathrm{~ms} ; 50 \mathrm{~ms}$ windows for 200-600 $\mathrm{ms} ; 100 \mathrm{~ms}$ windows for $600-1000 \mathrm{~ms}$ after cue). The timing of cue by hemisphere interaction effects closely replicate those found for the a priori time periods from the EEG literature, with two instances of small variations in offset after the end of the a priori windows, consistent with the amount of temporal variation reported across studies. During the middle period (a priori window $400-500 \mathrm{~ms}$ ), effects in the MFG and IPSa appear to continue until $550 \mathrm{~ms}$. Importantly, there are no isolated periods of attention-related effects occurring outside of the literature-derived a priori windows including the period from 550 to $800 \mathrm{~ms}$.

\section{Cross-talk issues}

While the spatial spread of activity created by the inverse method makes it more difficult to resolve activity in neighboring regions, the results illustrate how neighboring regions can be selectively identified when their activity differentially varies over time or as a function of experimental condition. For example, during 250$350 \mathrm{~ms}$, the LIP and cuneus have attention-related activity, whereas the IPSv does not, despite its location between LIP and cuneus (i.e., a neighbor to both regions). Even so, due to low spatial resolution, activity found in an ROI (e.g., LIP or cuneus) does not pinpoint the activity to that ROI nor restrict it to that ROI, but indicates that the activity arises in the vicinity of that ROI. In the present data, there are instances in which neighboring regions are active at the same time, however, because there are other times when they are independently active, we report all regions separately.

\section{Correspondence between direction-specific anticipatory attention activity and subsequent performance}

To determine whether these anticipatory attentional modulations of cortical activity benefit subsequent behavioral performance, we analyzed the magnitude of attentional modulation in each cortical region with respect to reaction time. Directionspecific attention modulation was calculated for fast and slow RTs and submitted to an ANOVA with two within-subjects fac- 
tors: RT (fast, slow) and hemisphere (left and right). Fast and slow response times were not significantly different for targets in left and right visual fields [fast, left visual field, $427.5 \pm 71.2 \mathrm{~ms}(\mathrm{SD})$; right visual field, $422.8 \pm 65.3 \mathrm{~ms}(\mathrm{SD})$; slow, left visual fields, $581.0 \pm 86.7 \mathrm{~ms}$ (SD); right visual field, $570.9 \pm 96.7 \mathrm{~ms}$ (SD)]. Results revealed a correspondence between attentional modulation during each time period during deployment and the speed of target detection, with the critical region differing for each period. In the first period (125-175 ms), the magnitude of attentional modulation in the cuneus region was greater for fast trials $\left(F_{(1,7)}=\right.$ $14.48, p<0.01,125-150 \mathrm{~ms})$. In the second period (250-350 ms) greater attentional modulation in FEF was associated with faster target detection $(F=7.26, p<$ $0.05,300-350 \mathrm{~ms})$. Greater attentional modulation in IPSv during the middle period (400-500 ms) corresponded with faster RT $(F=6.99, p<0.05,400-450 \mathrm{~ms})$. During the late period, there was an RT by hemisphere interaction in the LO region $\left(F_{(1,7)}=11.46, p<0.05,800-900 \mathrm{~ms} ; F=14.47, p<0.01\right.$, $900-1000 \mathrm{~ms}$ ), and post hoc tests revealed that this was due to the correspondence between attentional modulation and RT occurring predominantly in the left hemisphere.

The effects in FEF and IPSv indicate that these regions were an important part of the attentional systems at the early and middle periods, respectively, although they did not achieve significant direction-specific effects in the analysis above (Examination of multiple temporal periods during anticipatory deployment of visual spatial attention: direction-specific attention effectssection). Because large direction-specific amplitudes correspond with faster RTs and small amplitudes correspond with slower RTs in these areas, the combination across all trials is likely to reduce the overall direction-specific effect as analyzed above.

\section{Discussion}

The present study used cortical MEG ERF activity to identify cortical regions that show direction-specific activity during four time periods associated with anticipatory deployment of visual spatial attention.

It is widely postulated that cue-driven voluntary deployment of spatial attention must proceed via bottom-up extraction of symbolic cue meaning that leads to top-down direction-specific attentional deployment. However, there has not been a demonstration of the initial flow of activation from sensory to control regions before the earliest direction-specific modulation of sensory cortex. Such a modulation might indicate the time by which the symbolic cue meaning has been extracted and top-down attentional modulation of the sensory representation for the cued location initiated. In the present study, the temporal sequence of activity proceeds from occipital V1/V2 to extrastriate and parietal regions, then to frontal and then prefrontal cortex from 66 to 110 ms after cue (supplementary Fig. 1, available at www.jneurosci. org as supplemental material). The first direction-specific modulation in the cuneus occurs at 150-175 ms (and the strength of this modulation corresponds with faster subsequent target discrimination). The difference in timing between the bottom-up processing and the modulation of cuneus is sufficient for integra-
Direction Specific Attention Effect Magnitudes

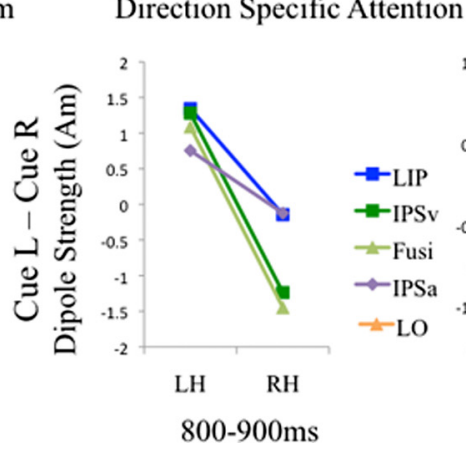

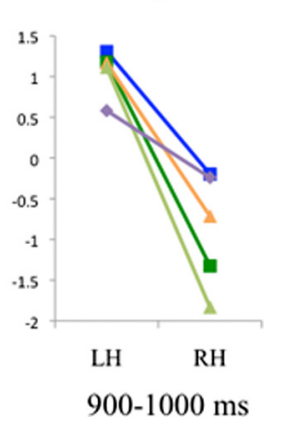

900-1000 ms

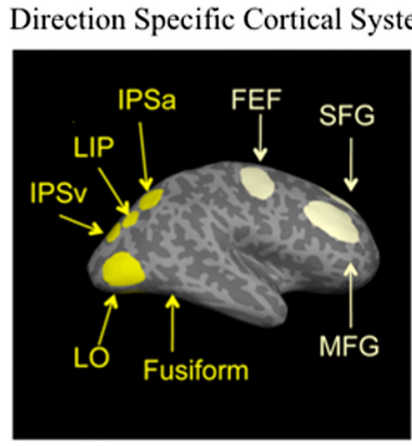

Figure 8. Late period ( $800-1000 \mathrm{~ms})$ : direction-specific attention system and frontal attention system. The final period during anticipatory attentional deployment leading up to the onset of the imperative stimulus (800-1000 ms after cue) involved two cortical systems. One system was related to direction-specific processes, and the other system was comprised of right hemisphere ral (fusiform) occipital regions, consistent with attention-related modulation based on both location and feature aspects of the anticipated target. In the frontal system, the right hemisphere frontal regions, the FEF, SFG and MFG, have significantly increased levels of activity during the final period of anticipatory attention.

tive processes in frontal and parietal control regions to occur and perhaps influence the direction-specific modulation of the cuneus, although current results cannot confirm a direct influence. The direction-specific modulation in cuneus could reflect an attention-related process or reentrant/secondary processing (Bar et al., 2006) related to extraction of the directional meaning or some type of late sensory processing of the slight physical asymmetry in the stimulus.

After the initial modulation of sensory cortex at 150-175 ms after cue, examination of three subsequent time periods during the cue-target delay revealed significant attention effects in different cortical regions. The next time period during attentional deployment (250-350 ms after cue) involves the FEF, LIP, cuneus, and possibly LO region, and may be similar in function to rapid covert shifts of spatial attention found in visual search. The task relevance of the left and right markers on the screen is ambiguous before the cue, but following the cue, one of the markers becomes relevant and can be targeted with a covert shift of attention. The magnitude of direction-specific activity in FEF during this early time period was predictive of faster reaction times, but when activity from FEF was averaged over trials with fast and slow response times, the overall attention effect was not statistically significant. Human fMRI studies have shown FEF, LIP, cuneus, and $\mathrm{LO}$ region to be involved in shifting visual spatial attention (Hopfinger et al., 2000; Corbetta and Shulman 2002; Molenberghs et al., 2007; Asplund et al., 2010), and the timing of activity in the current study suggests that FEF, LIP, cuneus (and possibly LO region) are involved in the covert targeting of the taskrelevant stimulus marker. This is consistent with the attentional control functions attributed to FEF and LIP from studies of covert shifts of attention (Colby and Goldberg 1999; Schall, 2002; Thompson et al., 2005; Buschman and Miller, 2009; Gregoriou et al., 2009) and with the top-down modulation of sensory regions by these control regions (Moore et al., 2003; Bressler et al., 2008). A burst of coherent gamma activity between widespread sensors over the head around this time in deployment (Doesburg et al., 2008) suggests that interactions between frontal, parietal, and sensory regions are involved. Consensus is building across studies of oscillatory and stimulus-locked ERF measures that attentional modulation can occur at a relatively early time in the deployment 
process (Green and McDonald, 2008; Siegel et al., 2008; Pantazis et al., 2009), and there is evidence for behavioral importance via correlations with performance in the superior parietal lobe (SPL), IPL (Green and McDonald, 2008), and LO region (Wyart and Tallon-Baudry, 2009), as well as from the relationship between FEF activity and performance in the present study.

Unlike the other periods during anticipatory attentional deployment, which show attention activity in control and sensory regions (suggesting top-down modulations of sensory representations), the middle period ( $400-500 \mathrm{~ms}$ ) involves solely frontal and parietal control regions (SFG, MFG, LIP, IPSa, IPL), suggesting a different function involving coordination between frontal and parietal control regions. This coordination, following covert targeting of the relevant location, may serve to integrate control processes needed to shift attention with respect to both the immediate spatial instruction (cue left/right) and the overall task instruction (discriminate the target feature at the cued location). Dynamics of direction-specific attention modulations in parietal and frontal regions have been shown in one other study, using oscillatory measures in the theta band, whereby activity peaked in frontal areas (MFG, SFG) in this time frame, but not in parietal regions (SPL, IPL) (Green and McDonald, 2008). Instead, parietal activity peaked during early (SPL, IPL) and late (IPL) periods. It may be that our stimulus-locked signal-averaged ERF measures pick up a transient and consistently stimulus-locked process needed to coordinate activity between parietal and frontal control regions under certain conditions. The involvement of direction-specific effects in parietal regions during this time period also appears to be frequency dependent, with the SPL, IPL, and IPS active in alpha and beta bands (Siegel et al., 2008; Pantazis et al., 2009), but not in theta or gamma bands (Green and McDonald, 2008; Siegel et al., 2008). The findings for this and the previous time period identify brain regions that contribute to the commonly reported scalp effects, EDAN and ADAN, occurring in these time periods (Hopf and Mangun, 2000; Nobre et al., 2000a; Praamstra et al., 2005; Green and McDonald, 2006; Jongen et al., 2006; van der Lubbe et al., 2006; Dale et al., 2008).

The late period from $800 \mathrm{~ms}$ after cue until target arrival involves direction-specific modulation of parietal (IPSa, IPSv, LIP) and occipital (LO, fusiform) regions, as well as non-directionspecific activity in right hemisphere frontal regions (FEF, SFG, MFG). While the function of these nonspecific effects in frontal areas is unclear from this study alone, results are consistent with a role for frontal cortex in sustaining visual spatial attention in general (Kastner and Ungerleider, 2000; Hopfinger et al., 2000; Vandenberghe et al., 2001; Grent-'t-Jong and Woldorff, 2007). The timing of coactivation with other regions suggests coordination with parietal control regions that are also thought to be involved in sustaining anticipatory visual spatial attention (Corbetta and Shulman, 2002; Silver et al., 2005; Serences and Yantis 2007; Saygin and Sereno 2008; Doesburg et al., 2009). This is also the only period in which both dorsal and ventral visual areas are modulated. Earlier time windows show attentional modulation only in dorsal sensory regions, suggesting primarily spatially related targeting, whereas the involvement of both dorsal and ventral regions in the final period leading up to target arrival suggests a more integrated biasing for space and feature. EEG/MEG studies have found posterior direction-specific anticipatory attention activity immediately preceding the imperative stimulus that may reflect a bias signal (Worden et al., 2000; Eimer et al., 2002; Praamstra et al., 2005; Sauseng et al., 2005; Slagter et al., 2005; Green and McDonald, 2006, 2010; Thut et al., 2006; van der Lubbe et al., 2006; Grent-'t-Jong and Woldorff, 2007; Dale et al.,
2008; for gamma-band evidence for decision bias, see also Wyart and Tallon-Baudry, 2009). There is some debate as to whether this late activity originates in parietal and/or occipital regions, and some studies have shown this activity to be related to performance, adding support for its role as a bias signal (Thut et al., 2006; Green and McDonald, 2008; Wyart and Tallon-Baudry, 2009). While the cortical origins are likely to depend in part on the task, recent studies, including the present one, now show such late activity, lasting until target arrival, in occipital regions (Green and McDonald, 2008) or both parietal and occipital regions (Siegel et al., 2008; Pantazis et al., 2009; present study), with performancerelated modulations found in occipital regions [inferior occipital gyrus (IOG) (Green and McDonald, 2008) and LO (present study)].

In summary, this study identifies direction-specific cortical ERF activity from multiple frontal, parietal, and sensory regions, adding to an emerging characterization of the complex electrophysiological signals arising from cortical regions dynamically involved in anticipatory deployment of spatial attention. The results show attention effects in different regions in each of the temporal periods examined during deployment. An early period involving FEF, LIP, cuneus, and possibly the LO region may reflect a covert shift to the cued location marker similar to a process found in visual search. Late attention effects in parietal and both dorsal and ventral occipital regions are consistent with the notion of a baseline shift/bias signal. A middle period involves only frontal and parietal regions, and might reflect coordination between control regions to transition from the early covert spatial shift to the subsequent biasing of both spatial and feature representations. Each of the temporal periods examined in cued attentional deployment is functionally important for performance, as indicated by a correspondence between reaction time and the magnitude of direction-specific attention activity in different regions during each period.

\section{References}

Asplund CL, Todd JJ, Snyder AP, Marois R (2010) A central role for the lateral prefrontal cortex in goal-directed and stimulus-driven attention. Nat Neurosci 13:507-512.

Bar M, Kassam KS, Ghuman AS, Boshyan J, Schmid AM, Dale AM, Hamalainen MS, Marinkovic K, Schacter DL, Rosen BR, Halgren E (2006) Top-down facilitation of visual recognition. Proc Natl Acad Sci U S A 103:449-454.

Bressler SL, Tang W, Sylvester CM, Shulman GL, Corbetta M (2008) Topdown control of human visual cortex by frontal and parietal cortex in anticipatory visual spatial attention. J Neurosci 28:10056-10061.

Brignani D, Lepsien J, Rushworth MF, Nobre AC (2008) The timing of neural activity during shifts of spatial attention. J Cog Neurosci 21:2369-2383.

Buschman TJ, Miller EK (2007) Top-down versus bottom-up control of attention in the prefrontal and posterior parietal cortices. Science 315:1860-1862.

Buschman TJ, Miller EK (2009) Serial, covert shifts of attention during visual search are reflected by the frontal eye fields and correlated with population oscillations. Neuron 63:386-396.

Capotosto P, Babiloni C, Romani GL, Corbetta M (2009) Frontoparietal cortex controls spatial attention through modulation of anticipatory alpha rhythms. J Neurosci 29:5863-5872.

Colby CL, Goldberg ME (1999) Space and attention in parietal cortex. Annu Rev Neurosci 23:319-349.

Corbetta M, Shulman GL (2002) Control of goal-directed and stimulusdriven attention in the brain. Nat Rev Neurosci 3:201-215.

Corbetta M, Akbudak E, Conturo TE, Snyder AZ, Ollinger JM, Drury HA, Linenweber MR, Petersen SE, Raichle ME, Van Essen DC, Shulman GL (1998) A common network of functional areas for attention and eye movements. Neuron 21:761-773.

Coull JT, Nobre AC (1998) Where and when to pay attention: the neural 
systems for directing attention to spatial locations and to time intervals as revealed by both PET and fMRI. J Neurosci 18:7426-7435.

Dale AM, Fischl B, Sereno MI (1999) Cortical surface-based analysis. I. Segmentation and surface reconstruction. Neuroimage 9:179-194.

Dale AM, Liu AK, Fischl BR, Buckner RL, Belliveau JW, Lewine JD, Halgren E (2000) Dynamic statistical parametric mapping: combining fMRI and MEG for high-resolution imaging of cortical activity. Neuron 26:55-67.

Dale CL, Simpson GV, Foxe JJ, Luks TL, Worden MS (2008) ERP correlates of anticipatory attention: spatial and non-spatial specificity and relation to subsequent selective attention. Exp Brain Res 188:45-62.

Darvas F, Pantazis D, Yildirim E, Leahy R (2004) Mapping human brain function with MEG and EEG: methods and validation. Neuroimage 23:S289-S299.

Desimone R, Duncan J (1995) Neural mechanisms of selective visual attention. Annu Rev Neurosci 18:193-222.

Doesburg SM, Roggeveen AB, Kitajo K, Ward LM (2008) Large-scale gamma-band phase synchronization and selective attention. Cereb Cortex 18:386-396.

Doesburg SM, Green JJ, McDonald JJ, Ward LM (2009) From local inhibition to long-range integration: a functional dissociation of alpha-band synchronization across cortical scales in visuospatial attention. Brain Res 1303:97-110.

Eimer M, van Velzen J, Driver J (2002) Cross-modal interactions between audition, touch and vision in endogenous spatial attention: ERP evidence on preparatory states and sensory modalities. J Cog Neurosci 14:254-271.

Fan J, Byrne J, Worden MS, Guise KG, McCandliss BD, Fossella J, Posner MI (2007) The relation of brain oscillations to attentional networks. J Neurosci 27:6197-6206.

Fischl B, Sereno MI, Dale AM (1999a) Cortical surface-based analysis. II: Inflation, flattening, and a surface-based coordinate system. Neuroimage 9:195-207.

Fischl B, Sereno MI, Tootell RB, Dale AM (1999b) High-resolution intersubject averaging and a coordinate system for the cortical surface. Hum Brain Mapp 8:272-284.

Fries P, Reynolds JH, Rorie AE, Desimone R (2001) Modulation of oscillatory neuronal synchronization by selective visual attention. Science 291:1560-1563.

Gitelman DR, Nobre AC, Parrish TB, LaBar KS, Kim Y, Meyer JR, Mesulam MM (1999) A large-scale distributed network for covert spatial attention. Brain 122:1093-1106.

Green JJ, McDonald JJ (2006) An event-related potential study of supramodal attentional control and crossmodal attention effects. Psychophysiology 43:161-171.

Green JJ, McDonald JJ (2008) Electrical Neuroimaging reveals timing of attentional control activity in human brain. PLoS Biol 6:731-738.

Green JJ, McDonald JJ (2010) The role of temporal predictability in the anticipatory biasing of sensory cortex during visuospatial shifts of attention. Psychophysiology 47:1057-1065.

Gregoriou GG, Gotts SJ, Zhou H, Desimone R (2009) High-frequency, long-range coupling between prefrontal and visual cortex during attention. Science 324:1207-1210.

Grent-'t-Jong T, Woldorff MG (2007) Timing and sequence of brain activity in top-down control of visual-spatial attention. PLoS Biol 5:114-126.

Halgren E, Boujon C, Clarke J, Wang C, Chauvel P (2002) Rapid distributed fronto-parietal-occipital processing stages during working memory in humans. Cereb Cortex 12:710-728.

Hämäläinen MS, Hari R, Ilmoniemi RJ, Knuutila J, Lounasmaa OV (1993) Magnetoencephalography - theory, instrumentation, and application to noninvasive studies of the working human brain. Rev Mod Phys 65:413-497.

Harter MR, Miller SL, Price NJ, LaLonde ME, Keyes AL (1989) Neural processes involved in directing attention. J Cogn Neurosci 1:223-237.

Hopf JM, Mangun GR (2000) Shifting visual attention in space: an electrophysiological analysis using high spatial resolution mapping. Clin Neurophysiol 111:1241-1257.

Hopfinger JB, Buonocore MH, Mangun GR (2000) The neural mechanisms of top-down attentional control. Nat Neurosci 3:284-291.

Jongen EM, Smulders FT, van Breukelen GJ (2006) Varieties of attention inn neutral trials: linking RT to ERPs and EEG frequencies. Psychophysiology 43:113-125.

Kastner S, Ungerleider LG (2000) Mechanisms of visual attention in the human cortex. Annu Rev Neurosci 23:315-341.
Kastner S, Pinsk MA, De Weerd P, Desimone R, Ungerleider LG (1999) Increased activity in human visual cortex during directed attention in the absence of visual stimulation. Neuron 22:751-761.

Kelly SP, Gomez-Ramirez M, Foxe JJ (2009) The strength of anticipatory spatial biasing predicts target discrimination at attended locations: a highdensity EEG study. Eur J Neurosci 30:2224-2234.

Lauritzen TZ, D’Esposito M, Heeger DJ, Silver MA (2009) Top-down flow of visual spatial attention signals from parietal to occipital cortex. J Vis 9:1-14.

Maunsell JH, Treue S (2006) Feature-based attention in visual cortex. Neurosci 9:317-322.

Meeren HK, Hadjikhani N, Ahlfors SP, Hamalainen MS, de Gelder B (2008) Early category-specific cortical activation revealed by visual stimulus inversion. PLoS One 3:1-11.

Molenberghs P, Mesulam MM, Peeters R, Vandenberghe RRC (2007) Remapping attentional priorities: differential contribution of superior parietal lobule and intraparietal sulcus. Cereb Cortex 17:2703-2712.

Moore T, Armstrong KM, Fallah M (2003) Visuomotor origins of covert spatial attention. Neuron 40:671-683.

Nobre AC, Sebestyen GN, Miniussi C (2000a) The dynamics of shifting visuospatial attention revealed by event-related potentials. Neuropsychologia 38:964-974.

Nobre AC, Gitelman DR, Dias EC, Mesulam MM (2000b) Covert visual spatial orienting and saccades: overlapping neural systems. Neuroimage $11: 210-216$.

Pantazis D, Simpson GV, Weber DL, Dale CL, Nichols TE, Leahy RM (2009) A novel ANCOVA design for analysis of MEG data with application to a visual attention study. Neuroimage 44:164-174.

Posner MI (1980) Orienting of attention. Q J Exp Psychol 32:3-25.

Praamstra P, Boutsen L, Humphreys GW (2005) Frontoparietal control of spatial attention and motor intention in human EEG. J Neurophysiol 94:764-774.

Ristic J, Friesen CK, Kingstone A (2002) Are eyes special? It depends on how you look at it. Psychonomic Bull Rev 9:507-513.

Rosen AC, Rao SM, Caffarra P, Scaglioni A, Bobholz JA, Woodley SJ, Hammeke TA, Cunningham JM, Prieto TE, Binder JR (1999) Neural basis of endogenous and exogenous spatial orienting: a functional MRI study. J Cog Neurosci 11:135-152.

Ruff CC, Bestmann S, Blankenburg F, Bjoertomt O, Josephs O, Weiskopf N, Deichmann R, Driver J (2008) Distinct causal influences of parietal versus frontal areas on human visual cortex: evidence from concurrent TMSfMRI. Cereb Cortex 18:817-827.

Saalmann YB, Pigarev IN, Vidyasagar TR (2007) Neural mechanisms of visual attention: how top-down feedback highlights relevant locations. Science 316:1612-1615.

Sauseng P, Klimesch W, Stadler W, Schabus M, Doppelmayr M, Hanslmayr S, Gruber WR, Birbaumer N (2005) A shift of visual spatial attention is selectively associated with human EEG alpha activity. Eur J Neurosci 22:2917-2926.

Saygin AP, Sereno MI (2008) Retinotopy and attention in human occipital, temporal parietal and frontal cortex. Cereb Cortex 18:2158-2168.

Schall JD (2002) The neural selection and control of saccades by the frontal eye field. Philos Trans R Soc Lond B Biol Sci 357:1073-1082.

Serences JT, Yantis S (2006) Selective visual attention and perceptual coherence. Trends Cogn Sci 10:38-45.

Serences JT, Yantis S (2007) Spatially selective representations of voluntary and stimulus-driven attentional priority in human occipital, parietal, and frontal cortex. Cereb Cortex 17:284-293.

Siegel M, Donner TH, Oostenveld R, Fries P, Engel AK (2008) Neuronal synchronization along the dorsal visual pathway reflects the focus of spatial attention. Neuron 60:706-719.

Silver MA, Ress D, Heeger DJ (2005) Topographic maps of visual spatial attention in human parietal cortex. J Neurophysiol 94:1358-1371.

Simpson GV, Dale CL, Luks TL, Miller WL, Ritter W, Foxe JJ (2006) Rapid targeting followed by sustained deployment of visual spatial attention. Neuroreport 17:1595-1599.

Slagter HA, Kok A, Mol N, Talsma D, Kenemans JL (2005) Generating spatial and nonspatial attentional control: an ERP study. Psychophysiology 42:428-439.

Thompson KG, Biscoe KL, Sato TR (2005) Neuronal basis of covert spatial attention in the frontal eye field. J Neruosci 25:9479-9487.

Thut G, Nietzel A, Brandt SA, Pascual-Leone A (2006) Alpha-band electro- 
encephalographic activity over occipital cortex indexes visuospatial attention bias and predicts visual target detection. J Neurosci 26:9494-9502.

van der Lubbe RH, Havik MM, Bekker EM, Postma A (2006) Taskdependent exogenous cuing effects depend on cue modality. Psychophysiology 43:145-160.

Vandenberghe R, Duncan J, Arnell K, Bishop S, Herrod N, Owen A, Minhas P, Dupont P, Pickard J, Orban G (2000) Maintaining and shifting attention within left or right hemifield. Cereb Cortex 10:706-713.

Vandenberghe R, Gitelman DR, Parrish TB, Mesulam MM (2001) Functional specificity of superior parietal mediation of spatial shifting. Neuroimage 14:661-673.

van Velzen J, Forster B, EImer M (2002) Temporal dynamics of lateralized
ERP components elicited during endogenous attentional shifts to relevant tactile events. Psychophysiology 39:874-878.

Worden MS, Foxe JJ, Wang N, Simpson GV (2000) Anticipatory biasing of visuospatial attention indexed by retinotopically specific alphaband electroencephalography increases over occipital cortex. J Neurosci 20:1-6.

Wyart V, Tallon-Baudry C (2009) How ongoing fluctuations in human visual cortex predict perceptual awareness: baseline shift versus decision bias. J Neurosci 29:8715-8725.

Yantis S, Schwarzbach J, Serences JT, Carlson RL, Steinmetz MA, Pekar JJ, Courtney SM (2002) Transient neural activity in human parietal cortex during spatial attention shifts. Nat Neurosci 5:995-1002. 